\section{Endodontic software for the $3 \mathrm{D}$ imaging era}

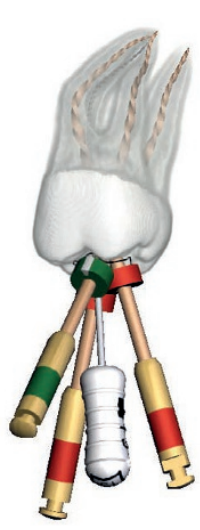

Always at the forefront of col-

laborative innovation and in line with their end-to-end solutions philosophy, Dentsply Sirona has now created a digitally integrated endodontic solution - 3D Endo.

3D Endo is the first CBCT software that allows endodontic treatment to be pre-planned and optimised using imaging data from Orthophos X-ray units, enabling the user to isolate the tooth being treated and locate the orifice and apex of the canals. This makes it possible to add more points to the computer image, resulting in precise tracking of each individual canal, no matter how curved it may be. The final $3 \mathrm{D}$ image can be rotated $360^{\circ}$, allowing the clinician to determine not only the working length, but also analyse the natural shape of the canal and select the appropriate files using the integrated file database.

3D Endo, in combination with Orthophos provides clinicians with the highest image quality at the lowest possible radiation dose resulting in better, safer, faster endodontic treatment and significantly improved patient communication.

To find out more visit www.3dendo. com, dentsplysirona.com or call us on 01932853422.

You can also access a range of education resources, video tutorials, courses and CPD webinars at dentsplysirona.com/ukeducation.

\section{An advocate for the profession}

When it comes to support and dedication, dental corporates and groups can rely on the Association of Dental Groups (ADG) to deliver.

That is because the ADG is a trade association that provides a nationwide support system for dental providers and employers (NHS and private) using a corporate or group model. The Association works in close collaboration with leading figures in the government and NHS as well as independent regulators and commissioners on a variety of issues, to ensure that members have the best

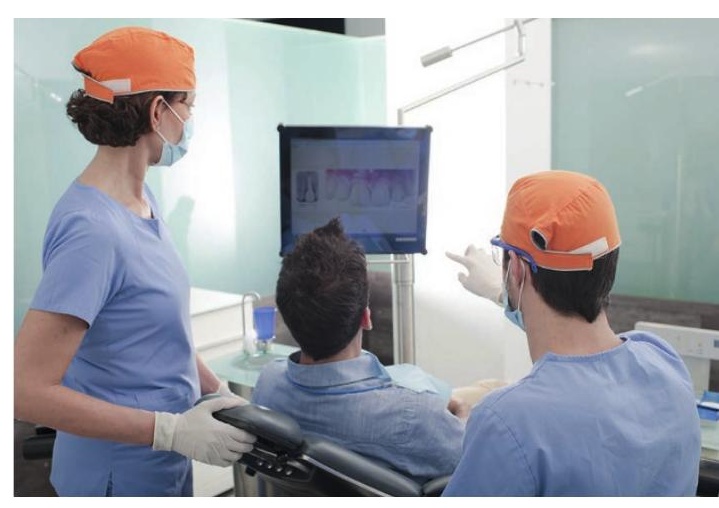
chance of delivering a high standard of care.

Along with its active campaigning and ongoing commitment to awarding dental students' hard work and ingenuity through its Bursary Awards, the ADG is a key advocate for the profession.

Moving forward, the ADG will continue to work hard to overcome barriers in dental care and ensure that dental corporates and groups can provide services to an even higher standard.

For more information about the ADG visit www.dentalgroups.co.uk

\section{Add value to your practice}

One of the three most important factors that patients value when choosing a dental practice is the use of advanced technology. Practices need to make patients aware of the modern equipment, technology and materials that are available to them, not just within the practice but also from the dental laboratory.

A custom developed, high performance polymer called Ultaire AKP is now available in the UK. Created by Solvay Dental 360 this innovative material is ideal for the fabrication of removable partial denture (RPD)

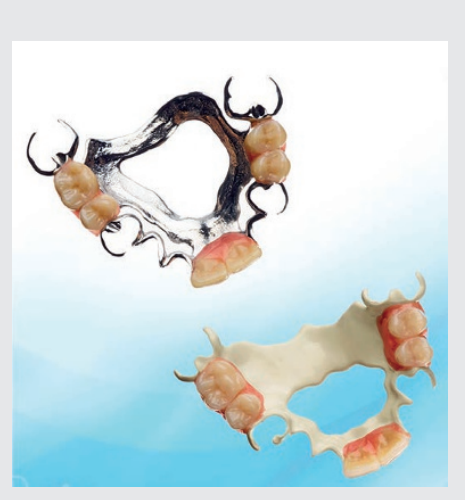
frames and offers patients a lightweight, comfortable and biocompatible alternative to metal.

Ultaire AKP has bone-like properties that many patients prefer and it provides both the elasticity and flexural strength to withstand loading. These frames are tooth supported, retentive and stable and offer a superior overall fit that could even limit future bone loss.

By presenting the high-tech option of Ultaire AKP, practices can add a valuable distinction to treatment recommendations and patient satisfaction.

For more information please visit www.solvaydental360.com.

\title{
Redefining corporate dentistry
}

As one of the fastest-growing UK dental corporates, Dental Partners is bringing a refreshing ethos to UK dentistry by building a supportive network for clinicians looking for the stability of a corporate position but without the constraints normally associated with this type of working environment.

When acquiring NHS and mixed practices, CEO of Dental Partners Neil Lloyd, looks, first and foremost, for a solid ethical focus and strong culture: 'I visit all the practices we buy myself and the first measure I take when I walk through the door is "Would I like to work here?" If the answer is no, I simply walk away?.

This new and energising perspective means that the relative newcomers to corporate dentistry are creating a new type of corporate environment, one that offers a stable support network for dental professionals looking for career development and a new challenge. Their aim is simple: to be the best place to work, and for dentists this ensures complete clinical freedom and a core emphasis on staff empowerment and personal development.

Good dentistry can only thrive in the right environment and Dental Partners believes that if it can make its practices the best place to work, by definition it will also be offering the best choice and best treatment to patients - putting dentistry first.

Find out more at www.dentalpartners. co.uk oremail contact@dentalpartners.co.uk. 